\title{
SEX-LINKAGE AND SEX-LIMITATION IN QUANTITATIVE INHERITANCE \\ II. DIALLEL CROSSES
}

\author{
R. J. KILLICK* \\ Department of Genetics, University of Birmingham, Birmingham B15 2TT, England \\ Received 4.iii.72
}

\begin{abstract}
SUMMARY
For animal breeders the diallel cross has one advantage over all other mating designs in that it permits sex-linkage to be distinguished from other causes of reciprocal differences. In other designs it is necessary to assume that other causes of reciprocal differences are absent (Killick, 1971). Since the transmission of $\mathrm{X}$ chromosomes to female progeny follows the same pattern as that of autosomes, sex-linkage can cause no disturbance to the data on female progeny; equally sex-linked inheritance cannot be distinguished from autosomal inheritance when data from female progeny alone are available. When data from male progeny are analysed alone, the factorial and Hayman analyses of variance are very informative. The $\left(W_{r}+V_{r}\right)$ and $\left(W_{r}-V_{r}\right)$ analyses of variance are undisturbed by sex-linkage, but if reciprocal crosses are averaged the regression line will not pass through the origin of the graph even with complete dominance. Provided reciprocal crosses are not averaged, all possible parameters may be estimated from the data. When data from male and female progeny are pooled the components of variance are more complex and less informative. The factorial analysis of variance is of little real help but the Hayman analysis of variance remains useful. The $W_{r} / V_{r}$ analyses retain their use but unless reciprocal crosses are averaged the $\left(W_{r}-V_{r}\right)$ analysis will be misleading. Not all the possible parameters can be estimated from the data. Thus the best approach will probably be to fit a simple model and then adjust and add to this until a satisfactory fit is obtained. Provided no more than 13 parameters are required, at least one degree of freedom will be available to test the adequacy of the model.
\end{abstract}

\section{INTRODUCTION}

THE diallel cross has been relatively rarely used by animal breeders and geneticists although Jinks (1954) and Dickinson and Jinks (1956) considered it to be a particularly economical method of investigating the inheritance of quantitiative traits in animals because the first filial generation alone is required. The familiar analyses of the diallel table assume inheritance to be autosomal; one drawback to the animal geneticist is therefore his uncertainty over the distortion that may result from the segregation of sexlinked genes. Nevertheless, various diallel analyses do allow the recognition of reciprocal differences as in Hayman's (1954) analysis of variance or the factorial analysis of Jinks and Broadhurst (1963). Durrant (1965) considered two types of mechanism causing reciprocal differences in diallel tables. These he called $\alpha$ inheritance, where the contribution of a gene in both homozygotes and heterozygotes differs in male and female arrays and $\beta$ inheritance where the dominance contribution of a gene differs in the two

\footnotetext{
* Present address: Scottish Plant Breeding Station, Pentlandfield, Roslin, Midlothian.
} 
reciprocal crosses. Weardon (1964) gave the expected mean squares obtainable from a diallel table assuming either maternal or reciprocal effects (as might arise from sex-linkage). In the latter model he did not distinguish between the sex of progeny, which makes for ambiguity. Topham (1966) provided an analysis of the diallel table to detect maternal effects and their interactions.

In this paper the effect of sex-linkage and sex-limitation on the analysis and interpretation of data from diallel crosses will be considered. In the present context sex-limitation implies a differential effect of the same autosomal gene in males and females. This may range from a slight quantitative difference to cases where the character is expressed in one sex only (e.g. milk yield in cattle).

\section{Model AND Parameters}

The diallel cross is constructed by crossing $n$ inbred lines in all possible combinations as both male and female parents to give $n^{2}$ families. These consist of $\frac{n^{2}-n}{2} \mathrm{~F}_{1}$ families, their $\frac{n^{2}-n}{2}$ reciprocal families and $n$ "selfed" families resulting from matings within the inbred lines. Where inbred lines are not available herds or breeds may be used; the bias caused by the heterozygosity will not be too serious provided the genetic differences within such parental groups are much less than those between them (Jinks, 1954).

The model and parameters describing sex-linkage used in this paper are those of Mather and Jinks (1971). A locus on the X chromosome having two alleles, $A$ and $a$ is considered to have the following effects:

$$
\begin{aligned}
& \text { Females Males } \\
& \begin{array}{ccccc}
A A & A a & a a & A & a \\
d_{x} & h_{x} & -d_{x} & d_{x}^{\prime} & -d_{x}^{\prime}
\end{array}
\end{aligned}
$$

Five parameters are required to specify the variation arising from sexlinked genes in randomly mating populations. These are:

$$
\begin{aligned}
& D_{R X}=\Sigma 4 u v\left[d_{x}+(v-u) h_{x}\right]^{2} \\
& H_{R X}=\Sigma 16 u^{2} v^{2} h_{x}^{2} \\
& D_{X}^{\prime}=\Sigma 4 u v d_{x}^{\prime 2} \\
& D_{X X}^{\prime}=\Sigma 4 u v d_{x} d_{x}^{\prime} \\
& F_{X X}^{\prime}=\Sigma 8 u v(u-v) d_{x}^{\prime} h_{x}
\end{aligned}
$$

With a parental population of inbred lines it is necessary to subdivide the $D_{R X}$ parameter further, so we define:

$$
\begin{aligned}
& D_{X}=\Sigma 4 u v d_{x}^{2} \\
& H_{1 X}=\Sigma 4 u v h_{x}^{2} \\
& H_{2 X}=\Sigma 16 u^{2} v^{2} h_{x}^{2} \quad\left(=H_{R X}\right) \\
& F_{X}=\Sigma 8 u v(u-v) d_{x} h_{x}
\end{aligned}
$$

For some analyses it is necessary to redefine the $F$ parameters to the form $\Sigma 4 u v d h$. This is indicated in the appropriate tables.

The model describing sex-limitation is that given by Killick (1971). The familiar three parameters which specify the mid-parent and the additive 
and dominance deviations of generation means $(m, d$ and $h)$ are considered to be increased by the quantities $f_{m}, f_{d}$ and $f_{h}$ respectively in females and decreased by the same amounts in males. If there is no sex-limitation, $f_{m}, f_{d}$ and $f_{h}$ are all zero and when sex-limitation is complete (i.e. only one sex expresses the character) $m=f_{m}, d=f_{d}$ and $h=f_{h}$. We define:

$$
\begin{array}{ll}
D=\Sigma 4 u v d^{2} & D_{S}=\Sigma 4 u v f_{d}^{2} \\
H_{1}=\Sigma 4 u v h^{2} & H_{1 S}=\Sigma 4 u v f_{h}^{2} \\
H_{2}=\Sigma 16 u^{2} v^{2} h^{2} & H_{2 S}=\Sigma 16 u^{2} v^{2} f_{h}^{2} \\
F=\Sigma 8 u v(u-v) d h & F_{S}=\Sigma 8 u v(u-v) f_{d} f_{h}
\end{array}
$$

The parameters on the left describe the autosomal genetic effects common to both sexes. Those on the right describe the sex-limited genetic effects.

Two different forms of analysis may be envisaged. With Method (a) male and female progeny are analysed separately so that each is treated as a separate experiment, while with Method $(b)$ male and female progeny are analysed jointly so that main effects and sex interactions are identified.

\section{Analyses}

(i) The factorial analysis of variance (Jinks and Broadhurst, 1963)

This is one of the simplest analyses of diallel tables and takes the form of a factorial analysis of variance identical to the North Carolina experi-

\begin{tabular}{|c|c|c|c|c|}
\hline \multicolumn{3}{|c|}{$\begin{array}{l}\begin{array}{l}\text { Sex-linkage } \\
\text { parameters }\end{array} \\
.\end{array}$} & \multicolumn{2}{|c|}{$\begin{array}{l}\text { Autosomal } \\
\text { parameters }\end{array}$} \\
\hline \multicolumn{2}{|c|}{ Females } & \multirow{2}{*}{$\begin{array}{c}\text { Males } \\
D_{X}^{\prime}\end{array}$} & \multicolumn{2}{|c|}{ Either sex } \\
\hline$D_{R X}$ & $H_{R X}$ & & $D_{R}$ & $H_{R}$ \\
\hline$\frac{1}{4}$ & - & 1 & $\frac{1}{4}$ & - \\
\hline$\frac{1}{4}$ & - & - & 1 & - \\
\hline 一 & $\frac{1}{4}$ & - & - & $\frac{1}{4}$ \\
\hline- & - & - & - & - \\
\hline
\end{tabular}
ment II of Comstock and Robinson (1952), so the variation is partitioned as in tables 1 and 2 of Killick (1971). The genetical interpretations of the components of variance from such an analysis of the diallel cross are shown in table 1 .

TABLE 1

Components of variance for the factorial analysis of variance $\operatorname{Method}(a)$, i.e. sexes analysed separately

Method $(b)$, i.e. sexes pooled

Sex-linkage parameters

$\begin{array}{lrrrrr} & \overbrace{R X} & D_{X}^{\prime} & D_{X X}^{\prime} & H_{R X} & F_{X X}^{\prime} \\ \sigma_{f}^{2} & \frac{1}{16} & \frac{1}{4} & \frac{1}{4} & - & -\frac{1}{8} \\ \sigma_{m}^{2} & 16 & - & - & - & - \\ \sigma_{m f}^{2} & - & - & - & 16 & - \\ \sigma_{s f}^{2} & \frac{1}{16} & \frac{1}{4} & -\frac{1}{16} & - & \frac{1}{8} \\ \sigma_{s m}^{2} & 1 & - & - & - & - \\ \sigma_{s m f}^{2} & - & - & - & \frac{1}{16} & - \\ \sigma_{w}^{2} & - & - & - & - & -\end{array}$

Autosomal parameters

$\begin{array}{cccc}D_{R} & H_{R} & D_{R S} & H_{R S} \\ 4 & - & - & - \\ 4 & - & - & - \\ - & 1 & - & - \\ - & - & \frac{1}{4} & - \\ - & - & 4 & - \\ - & - & - & 4 \\ - & - & - & -\end{array}$


Method (a)

The transmission of $X$ chromosomes to female progeny follows exactly the same pattern as the transmission of autosomes. Consequently the effects of the sex chromosomes cannot be separated from those of the autosomes, but equally sex-linkage cannot distort the analysis of female data. The additive and dominance effects of the combined autosomes and sex chromosomes can be separated since

and

$$
\sigma_{f}^{2}=\sigma_{m}^{2}=\frac{1}{4}\left(D_{R X}+D_{R}\right)
$$

$$
\sigma_{m f}^{2}=\frac{1}{4}\left(H_{R X}+H_{R}\right)
$$

where the subscripts $f, m$ and $m f$ indicate the component of variance for female and male parent arrays and their interaction respectively. With unequal allele frequencies the additive parameters contain some dominance variation. If $\sigma_{f}^{2} \neq \sigma_{m}^{2}$ the presence of some mechanism, other than sexlinkage, causing reciprocal differences may be detected. No other design permits this distinction to be made; in all the designs used for the analysis of randomly mating base populations, it is necessary to assume that sexlinkage alone is responsible for reciprocal differences (Killick, 1971).

Sex-linkage in male progeny causes $\sigma_{f}^{2}$ to exceed $\sigma_{m}^{2}$. It is possible, therefore, to differentiate between the additive effect of the hemizygous $\mathrm{X}$ chromosome, the additive effect of the autosomes and the dominance effects of the autosomes by estimating the parameters representing each of these effects.

\section{$\operatorname{Method}(b)$}

The expectation of the component of variance for sexes, $\sigma_{g}^{2}$, is not given in table 1 as it is a fixed effect, not a true variance, having a complex interpretation not definable in terms of the parameters used in this paper. $\sigma_{s}^{2}$ will therefore be ignored in this and in subsequent analyses. This leaves six statistics from which nine parameters are to be estimated, so clearly full separation of the various effects is impossible. However, various combinations of the components do permit estimation of a few parameters. For example:

$$
\begin{aligned}
& \left(\sigma_{f}^{2}-\sigma_{m}^{2}\right)-\left(\sigma_{s f}^{2}-\sigma_{s m}^{2}\right)=\frac{1}{2} D_{X X}^{\prime}-\frac{1}{4} F_{X X}^{\prime} \\
& \left(\sigma_{f}^{2}-\sigma_{m}^{2}\right)+\left(\sigma_{\delta f}^{2}-\sigma_{s m}^{2}\right)=\frac{1}{2} D_{X}^{\prime}
\end{aligned}
$$

but the standard errors of such estimates are bound to be large. Similarly $\left(\sigma_{m f}^{2}-\sigma_{s m f}^{2}\right)=\frac{1}{4}\left(H_{R}-H_{R S}\right)$, an estimate of the degree of autosomal dominance, less the sex-limited dominance. Nevertheless, the main use of the factorial analysis of variance is the establishment of the significance of genetic effects rather than their estimation.

(ii) The analysis of variance of Hayman (1954)

Four main sources of variation are recognised in Hayman's analysis of variance. These, with their autosomal interpretations, are:

"a" item additive genetical variation

"b" item non-additive genetical variation subdivided into three parts 
" $\mathrm{b}_{1}$ " item overall potence

" $\mathrm{b}_{2}$ " item potence due to particular parents

" $\mathrm{b}_{3}$ " item residual non-additive variation

"c" item reciprocal differences attributable to particular parents

" $d$ " item reciprocal differences not attributable to particular parents.

No distinction is made as to the origin of the reciprocal differences measured by the " $c$ " and " $d$ " items. Such a distinction can only be deduced from inspection of the data and not from the analysis of variance. The Hayman analysis was not intended for the estimation of parameters and is not suitable for this purpose. Its advantage lies in detecting significant genetical effects.

\section{Method (a)}

The expectations for the mean squares in the Hayman analysis are shown in table 2. When female progeny are analysed alone, each item

TABLE 2

Interpretation of the Hayman analysis of variance. Each mean square is a function of the following parameters

Method (a), i.e. sexes separate

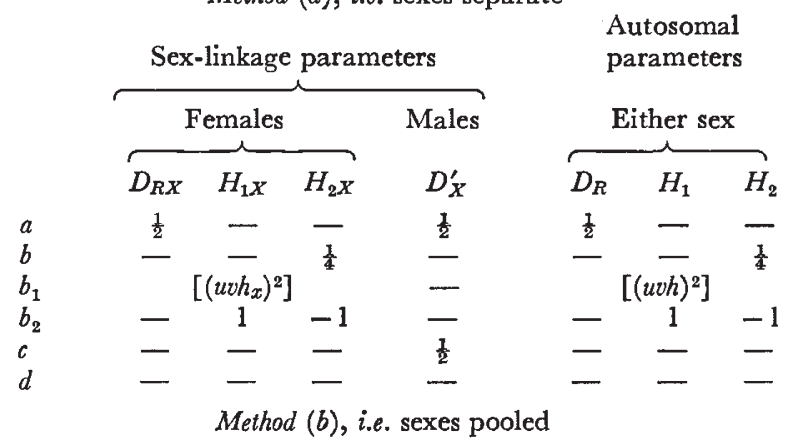

Sex-linkage parameters

\begin{tabular}{|c|c|c|c|c|c|c|c|c|c|c|c|c|}
\hline & & & & & & \multirow{2}{*}{\multicolumn{2}{|c|}{$H_{2 S}$}} \\
\hline & $D_{R X}$ & $H_{1} X$ & $H_{2 X}$ & $D_{X}^{\prime}$ & $D_{X X}^{\prime}$ & $F_{X X}^{\prime}$ & $D_{R}$ & $H_{1}$ & $H_{2}$ & $D_{R S}$ & & \\
\hline$a$ & $\frac{1}{8}$ & - & - & $\frac{1}{8}$ & $\frac{1}{4}$ & $-\frac{1}{8}$ & $\frac{1}{8}$ & - & - & - & - & - \\
\hline$b$ & - & - & $\frac{1}{16}$ & - & - & - & - & - & $\frac{1}{16}$ & - & - & - \\
\hline$b_{1}$ & & & {$\left[\left(\frac{1}{2} u r\right.\right.$} & $\left.\left.h_{x}\right)^{2}\right]$ & & & & & {$\left[\left(\frac{1}{2}\right)\right.$} & $\left.h)^{2}\right]$ & & \\
\hline$b_{2}$ & - & $\frac{1}{4}$ & $-\frac{1}{4}$ & - & - & - & - & $\frac{1}{4}$ & $-\frac{1}{4}$ & - & - & - \\
\hline$c$ & - & - & - & $\frac{1}{8}$ & - & - & - & - & - & - & - & - \\
\hline$d$ & - & - & - & - & - & - & - & - & - & - & - & - \\
\hline$S \times a$ & $\frac{1}{8}$ & - & - & $\frac{1}{8}$ & $-\frac{1}{4}$ & $\frac{1}{8}$ & - & - & - & $\frac{1}{8}$ & - & - \\
\hline$S \times b$ & - & - & $\frac{1}{16}$ & - & - & - & - & - & - & - & - & $\frac{1}{16}$ \\
\hline$S \times b_{1}$ & & & {$\left[\left(\frac{1}{2} u r\right.\right.$} & $\left.\left.h_{x}\right)^{2}\right]$ & & & & & {$\left[\left(\frac{1}{2} u\right.\right.$} & $\left.\left(f_{h}\right)^{2}\right]$ & & \\
\hline$S \times b_{2}$ & - & $\frac{1}{4}$ & $-\frac{1}{4}$ & - & - & - & - & - & - & - & $\frac{1}{4}$ & $-\frac{1}{4}$ \\
\hline$S \times c$ & - & - & - & $\frac{1}{8}$ & - & - & 一 & - & - & - & - & - \\
\hline$S \times d$ & - & - & - & - & - & - & - & - & - & - & - & - \\
\hline
\end{tabular}

continues to detect the effects as described above, whether the source is autosomal or sex-linked. Consequently, sex-linkage has no disturbing influence on the analysis. When male progeny are analysed alone, the additive effect of the sex chromosome makes an equal contribution to the " a " and the " c " items. The " b" item is unaffected since the hemizygous 
$\mathrm{X}$ chromosome cannot exhibit dominance. The interpretation of the analysis remains valid since the "a " item detects additive gene action and the "c" item detects reciprocal differences. As with the factorial analysis of variance, this provides an unambiguous test for sex-linkage since if the " c " item is significant for male progeny but not for female progeny, then sex-linkage alone must be responsible. The difference between the "a" and " $c$ " mean squares for male progeny indicates the relative proportions of additive gene effects on the autosomes and sex-chromosome.

\section{Method (b)}

With the exception of the "a" item all the main effects and their sex interactions in the analysis have the same expectation for sex-linked genes; differences between the main effects and their sex-interactions can be ascribed to autosomal variation (see table 2). It will be seen that each item continues to detect the type of gene action conventionally associated with it but the main effects are now compounded of sex-linked and autosomal effects while the sex interactions are compounded of sex-linked and sex-limited effects.

\section{(iii) Variances and covariances of arrays (Finks, 1954)}

Jinks (1954) showed that in the absence of epistasis the difference between the covariance of array family means on their non-recurrent parents, $W_{r}$, and the variance of the array family means, $V_{r}$, should be constant for all arrays. The regression of $W_{r}$ on $V_{r}$ should be a line of unit slope with the most dominant parents giving rise to array points nearest the origin of the graph and the most recessive parents giving rise to array points furthest from the origin. If the line passes through the origin, dominance is complete; otherwise the distance from the origin to the point where the line intersects the $W_{r}$ axis is equal to $\frac{1}{4}\left(D-H_{1}\right)$. If the point of intersection is on the positive side of the origin, dominance is incomplete, and if it is on the negative side, overdominance is to be suspected. If there is no dominance, all the array points centre at a single value of

$$
V_{r}=\frac{1}{2} W_{r}=\frac{1}{4} V_{p}
$$

The significance of the dominance variation is shown by variation in values of $\left(W_{r}+V_{r}\right)$ for each array (Allard, 1956). The absence of epistasis and of correlated gene distributions, and the presence of dominance, are conveniently tested by an analysis of variance of $\left(W_{r}-V_{r}\right)$ and $\left(W_{r}+V_{r}\right)$. values using their block interactions as error (Mather and Jinks, 1971). It is usual to average reciprocal crosses before computing values of $W_{r}$ and $V_{r}$, but here the expectations will be given for the data both before and after averaging over reciprocal crosses.

\section{Method (a)}

The expectations of the variances and covariances when sexes are analysed separately are shown in table 3 . The expectations of the sexlinkage parameters in female progeny are identical to those of the autosomal parameters so, as might be expected, sex-linkage cannot distort any $W_{r}, V_{r}$ analysis from female data; the interpretation covers both sex-linked and autosomal genes. The situation is a little different in male progeny. When the data are averaged over reciprocals, sex-linked genes contribute $\frac{1}{4} D_{X}^{\prime}$ 
to the value of $\left(W_{r}-V_{r}\right)$ and $\frac{3}{4} D_{X}^{\prime}$ to the value of $\left(W_{r}+V_{r}\right)$ for every array. Sex-linked genes therefore contribute nothing to the variance of arrays in either the $\left(W_{r}+V_{r}\right)$ or the $\left(W_{r}-V_{r}\right)$ analysis of variance and so do not bias the results. When the data are not averaged over reciprocal crosses the only contribution sex-linked genes make is $2 D_{x}^{\prime}$ to $\left(W_{r \sigma^{z}}+V_{r \sigma^{z}}\right)$ for every array, so here again, provided male and female parent arrays are considered separately, sex-linkage causes no bias to the results. $D_{X}^{\prime}$ is estimated simply as half the average difference between

$$
\left(W_{r \sigma^{3}}+V_{r \sigma^{3}}\right)-\left(W_{r q}+V_{r o}\right)
$$

for all arrays. The effect of sex-linkage may be separated from other causes of reciprocal differences in that it causes a difference between the statistics

TABLE 3

Variances and covariances of arrays

Method (a), i.e. sexes separate

$\overbrace{D_{X} H_{1 X} \quad F_{X}}^{\text {Females }} D_{X}^{\prime} \overbrace{D_{D} H_{1} \quad F}^{\text {Males }}$

Not averaged over reciprocal crosses:

\begin{tabular}{|c|c|c|c|c|c|c|}
\hline$V$ 여 $A A$ & $\frac{1}{4}$ & $\frac{1}{4}$ & $-\frac{1}{2}$ & - & $\frac{1}{4}$ & $\frac{1}{4}$ \\
\hline$V$ ㅇ $a a$ & $\frac{1}{4}$ & $\frac{1}{4}$ & $\frac{1}{2}$ & - & $\frac{1}{4}$ & $\frac{1}{4}$ \\
\hline$V_{\delta} A$ & $\frac{1}{4}$ & $\frac{1}{4}$ & $-\frac{1}{2}$ & 1 & $\frac{1}{4}$ & $\frac{1}{4}$ \\
\hline$V$ ชै $a$ & $\frac{1}{4}$ & $\frac{1}{4}$ & $\frac{1}{2}$ & 1 & $\frac{1}{4}$ & $\frac{1}{4}$ \\
\hline$W$ 우 $A A$ & $\frac{1}{2}$ & - & $-\frac{1}{2}$ & - & $\frac{1}{2}$ & - \\
\hline$W$ o $a a$ & $\frac{1}{2}$ & - & $\frac{1}{2}$ & - & $\frac{1}{2}$ & - \\
\hline$W \overbrace{}^{\star} A$ & $\frac{1}{2}$ & - & $-\frac{1}{2}$ & 1 & $\frac{1}{2}$ & - \\
\hline$W \sigma^{\pi} a$ & & - & $\frac{1}{2}$ & 1 & $\frac{1}{2}$ & - \\
\hline
\end{tabular}

Averaged over reciprocal crosses:

$$
\begin{array}{lllllllr}
V A A & \frac{1}{4} & \frac{1}{4} & -\frac{1}{2} & \frac{1}{4} & \frac{1}{4} & \frac{1}{4} & -\frac{1}{2} \\
V a a & \frac{1}{4} & \frac{1}{2} & \frac{1}{2} & \frac{1}{4} & \frac{1}{4} & \frac{1}{4} & \frac{1}{2} \\
W A A & \frac{1}{2} & - & -\frac{1}{2} & \frac{1}{2} & \frac{1}{2} & - & -\frac{1}{2} \\
W a a & \frac{1}{2} & - & \frac{1}{2} & \frac{1}{2} & \frac{1}{2} & - & \frac{1}{2} \\
& * F_{X}=\Sigma 4 u v d_{x} h_{x} . & F=\Sigma 4 u v d h .
\end{array}
$$

from male and female parent arrays in male progeny alone; other causes of reciprocal differences would create similar differences in female progeny also. Sex-linkage in the averaged male data will prevent the $W_{r}, V_{r}$ regression line passing through the origin of the graph since the intercept will be $\frac{1}{4}\left(D-H_{1}+D_{X}^{\prime}\right)$ units from the origin and this will be positive even if all the autosomal genes are completely dominant. When reciprocal crosses are not averaged, and male and female parent arrays are considered separately, this effect on the intercept will not occur. In such cases

for all arrays.

$$
\left(W_{r q}-V_{r q}\right)=\left(W_{r \sigma^{z}}-V_{r \sigma^{3}}\right)=\frac{1}{4}\left(D-H_{1}\right)
$$

Method (b)

The expectations of array variances and covariances when male and female progeny are analysed jointly are shown in table 4 . Considering 
firstly the case where reciprocal families are averaged prior to computation of these statistics it can be seen that sex-linkage distorts neither the $\left(W_{r}-V_{r}\right)$ nor the $\left(W_{r}+V_{r}\right)$ analyses since

$\left(W_{A A}-V_{A A}\right)=\left(W_{a a}-V_{a a}\right)$ and $\left(S \times W_{A A}-S \times V_{A A}\right)=\left(S \times W_{a a}-S \times V_{a a}\right)$, where $W_{A A}$ is the covariance of an array, the recurrent parent of which has

TABLE 4

Variances and covariances of arrays

Method $(b)$, i.e. sexes pooled

Sex-linkage parameters*

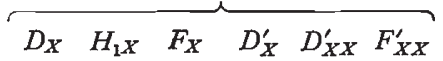

Autosomal parameters*

Not averaged over reciprocal crosses:

\begin{tabular}{|c|c|c|c|c|c|c|c|c|c|c|c|c|}
\hline$V$ ㅇ $A A$ & $\frac{1}{16}$ & $\frac{1}{16}$ & $-\frac{1}{8}$ & - & 一 & $\rightarrow$ & $\frac{1}{4}$ & $\frac{1}{4}$ & $-\frac{1}{2}$ & $\ldots$ & - & - \\
\hline$V ㅇ a ~ a a$ & $\frac{1}{16}$ & $\frac{1}{16}$ & & - & - & - & $\frac{1}{4}$ & $\frac{1}{4}$ & $\frac{1}{2}$ & 一 & - & - \\
\hline$V \hat{\delta} A$ & $\frac{1}{16}$ & $\frac{1}{16}$ & $-\frac{1}{8}$ & $\frac{1}{4}$ & $\frac{1}{4}$ & $-\frac{1}{4}$ & $\frac{1}{4}$ & $\frac{1}{4}$ & $-\frac{1}{2}$ & - & - & - \\
\hline$V \widehat{\partial} a$ & $\frac{1}{16}$ & $\frac{1}{16}$ & $\frac{1}{8}$ & $\frac{1}{4}$ & $\frac{1}{4}$ & & $\frac{1}{4}$ & $\frac{1}{4}$ & $\frac{1}{2}$ & - & - & - \\
\hline$W$ W $A A$ & $\frac{1}{8}$ & - & $-\frac{1}{8}$ & 一 & $\frac{1}{8}$ & $-\frac{1}{8}$ & $\frac{1}{2}$ & 一 & $-\frac{1}{2}$ & - & - & - \\
\hline$W$ Woa & $\frac{1}{8}$ & 一 & $\frac{1}{8}$ & - & $\frac{1}{8}$ & $\frac{1}{8}$ & $\frac{1}{2}$ & - & $\frac{1}{2}$ & - & - & - \\
\hline$W \delta$ & $\frac{1}{8}$ & - & $-\frac{1}{8}$ & $\frac{1}{4}$ & $\frac{3}{8}$ & $-\frac{1}{8}$ & $\frac{1}{2}$ & - & $-\frac{1}{2}$ & - & - & -0 \\
\hline Wठ $a$ & $\frac{1}{8}$ & 一 & $\frac{1}{8}$ & $\frac{1}{4}$ & $\frac{3}{8}$ & $\frac{1}{8}$ & $\frac{1}{2}$ & - & $\frac{1}{2}$ & - & 一 & - \\
\hline$S V O A A$ & $\frac{1}{16}$ & $\frac{1}{16}$ & $-\frac{1}{8}$ & - & - & - & - & - & - & $\frac{1}{4}$ & $\frac{1}{4}$ & $-\frac{1}{2}$ \\
\hline$S V$ So $a a$ & $\frac{1}{16}$ & $\frac{1}{16}$ & $\frac{1}{8}$ & - & 一 & - & $一$ & - & - & $\frac{1}{4}$ & $\frac{1}{4}$ & \\
\hline$S V ठ A$ & $\frac{1}{16}$ & $\frac{1}{16}$ & $-\frac{1}{8}$ & $\frac{1}{4}$ & $-\frac{1}{4}$ & $\frac{1}{4}$ & - & - & - & $\frac{1}{4}$ & $\frac{1}{4}$ & $-\frac{1}{2}$ \\
\hline$S V$ ठै $a$ & $\frac{1}{16}$ & $\frac{1}{16}$ & $\frac{1}{8}$ & $\frac{1}{4}$ & $-\frac{1}{4}$ & $-\frac{1}{4}$ & - & - & - & $\frac{1}{4}$ & $\frac{1}{4}$ & \\
\hline$S W$ 우 $A A$ & $\frac{1}{8}$ & - & $-\frac{1}{8}$ & $\longrightarrow$ & $-\frac{1}{8}$ & $\frac{1}{8}$ & - & - & - & $\frac{1}{2}$ & - & $-\frac{1}{2}$ \\
\hline SWㅇa & $\frac{1}{8}$ & - & $\frac{1}{8}$ & - & $-\frac{1}{8}$ & $-\frac{1}{8}$ & - & 一 & - & $\frac{1}{2}$ & - & \\
\hline$S W \precsim A$ & $\frac{1}{8}$ & - & $-\frac{1}{8}$ & $\frac{1}{4}$ & $-\frac{3}{8}$ & $\frac{1}{8}$ & 一 & - & $一$ & $\frac{1}{2}$ & - & $-\frac{1}{2}$ \\
\hline$S W \delta a$ & $\frac{1}{8}$ & - & $\frac{1}{8}$ & $\frac{1}{4}$ & $-\frac{3}{8}$ & $-\frac{1}{8}$ & - & 一 & - & $\frac{1}{2}$ & - & $\frac{1}{2}$ \\
\hline
\end{tabular}

Averaged over reciprocal crosses:

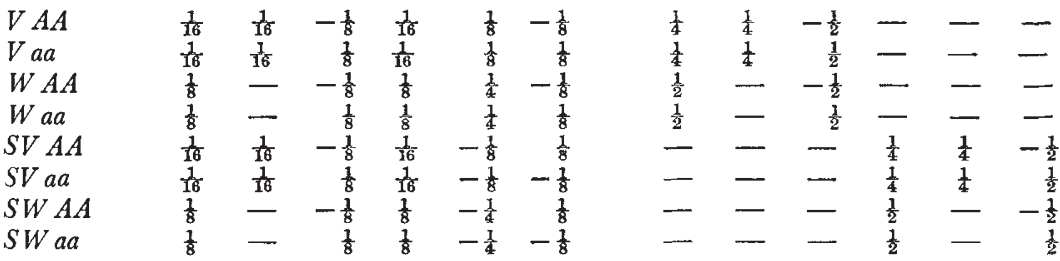

$$
\begin{aligned}
& * F_{X X}^{\prime}=\Sigma 4 u v d_{x}^{\prime} h_{x} . \quad F_{X}=\Sigma 4 u v d_{x} h_{x} . \quad F=\Sigma 4 u v d h . \quad F_{S}=\Sigma 4 u v f_{d} f_{h} .
\end{aligned}
$$

the increasing allele etc., i.e. the difference between the covariance and variance of every array is constant. Similarly

$$
\left(W_{A A}+V_{A A}\right)-\left(W_{a a}+V_{a a}\right)=-\frac{1}{2} F_{X}-\frac{1}{2} F_{X X}^{\prime}-F
$$

so the dominance of both sex chromosomes and autosomes contributes to the variation between arrays. If the values of the corresponding sex interactions are considered, the effect of $F_{X}$ opposes that of $F_{X X}^{\prime}$ and the analysis will be less effective since

$$
\left(S \times W_{A A}+S \times V_{A A}\right)-\left(S \times W_{a a}+S \times V_{a a}\right)=-\frac{1}{2} F_{X}+\frac{1}{2} F_{X X}^{\prime}-F_{S} .
$$

Other combinations of statistics can be derived to test the significance of dominance. For example, the variation in values of $\left(W_{r}+S \times W_{r}\right)$ may be used since $\left(W_{A A}+S \times W_{A A}\right)-\left(W_{a a}+S \times W_{a a}\right)=\frac{1}{2} F_{X}-F-F_{S}$. 
Even with complete autosomal dominance the $W_{r}, V_{r}$ regression line will not pass through the origin of the graph but cut the $W_{r}$ axis at a point $\frac{1}{16} D_{X}^{\prime}+\frac{1}{8} D_{X X}^{\prime}$ from it. The $S \times W_{r}, S \times V_{r}$ regression line will cut the $S \times W_{r}$ axis at $\frac{1}{16} D_{X}^{\prime}-\frac{1}{8} D_{X X}^{\prime}$.

In cases where reciprocal families are not averaged prior to computing the statistics a different situation obtains. The analysis of variance of $\left(W_{r}-V_{r}\right)$ using either male or female parent arrays, and either the main effects or sex interactions can not be interpreted in the normal way. This is because $F_{X X}^{\prime}$ contributes to the variation between arrays which cannot therefore be ascribed reliably to epistasis or correlated gene distributions. The $\left(W_{r}+V_{r}\right)$ analysis is quite satisfactory when performed on either male parent or female parent arrays, and detects both sex-linked and autosomal dominance. The contribution of $F_{X X}^{\prime}$ to the variation between male parent arrays is three times that in female parent arrays. The former are therefore more efficient in detecting sex-linked dominance.

\section{(iv) Estimation of parameters}

Whilst some parameters may be estimated from the factorial analysis of variance the method of estimation described by Jinks (1954) is more efficient. Four statistics are used; mean variance within arrays, $V_{r}$; variance of array means, $V_{\tilde{r}}$; variance of parents, $V_{p}$; and the covariance of parents and offspring, $\bar{W}_{r}$. Jinks states that the last statistic may be calculated in either of two ways; as the mean of the covariance of array members on their non-recurrent parents or as the covariance of array means on the recurrent parent of each array. However, this identity applies only to symmetrical tables as when reciprocal crosses are averaged prior to computation and does not apply when reciprocal differences exist. In the latter case the mean covariance of members of female parent arrays on their non-recurrent (i.e. male) parents is algebraically equivalent to the covariance of male parent array means on the recurrent parent of those arrays. To avoid any confusion reference will only be made to the covariance of array means on the recurrent parent of the arrays designated $W_{r \text { q }}$ for female parent array means and $W_{r \delta}$ for male parent array means.

\section{Method (a)}

The coefficients of the sex-linkage parameters for female progeny are identical to the corresponding autosomal parameters whether the data are averaged over reciprocal crosses or not (see table 5). Consequently no separation of sex-linked and autosomal parameters is possible, but their joint effects can be estimated. Because the expectations of male parent arrays are the same as those of female parent arrays the data must be averaged over reciprocal crosses to facilitate estimation of these parameters. For male progeny $D_{X}^{\prime}$ is completely correlated with $D$ in the averaged data, so these two parameters cannot be separated. When the data are not averaged over reciprocals all five parameters can be estimated.

\section{Method (b)}

Table 6 shows the expectations of statistics when the sexes of progeny are combined. When the data are averaged over reciprocal crosses before computation of the statistics, only eight statistics can be derived although 
TABLE 5

Expectation of statistics from a diallel table

Method (a), i.e. sexes separate

Sex-linkage parameters

Autosomal parameters

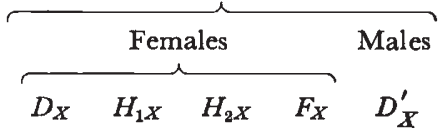

Either sex

$\overbrace{D H_{1} \quad H_{2} \quad F}$

Averaged over reciprocal crosses:

$$
\begin{aligned}
& \begin{array}{l}
V_{p} \\
V_{r} \\
V_{\dot{r}} \\
W_{r}
\end{array} \\
& \begin{array}{lllll}
1 & - & - & - & 1 \\
\frac{1}{4} & \frac{1}{1} & - & -\frac{1}{4} & 4 \\
\frac{1}{4} & \frac{1}{4} & -\frac{1}{4} & -\frac{1}{4} & \frac{1}{4} \\
\frac{7}{2} & - & - & -\frac{1}{4} & \frac{1}{2}
\end{array} \\
& \begin{array}{llll}
1 & - & - & - \\
\frac{1}{4} & 4 & - & -1 \\
\frac{1}{4} & 4 & -1 & -\frac{1}{4} \\
\frac{1}{2} & - & - & -\frac{1}{4}
\end{array}
\end{aligned}
$$

\begin{tabular}{|c|c|c|c|c|c|}
\hline$V_{p}$ & 1 & - & - & - & 1 \\
\hline $\bar{V}_{r q}$ & $\frac{1}{4}$ & 1 & - & $-\frac{1}{4}$ & - \\
\hline $\begin{array}{l}V_{r o s} \\
V_{* 0}\end{array}$ & $\frac{1}{1}$ & $\$$ & - & -4 & 1 \\
\hline $\begin{array}{l}\dot{r}_{\dot{q}} \\
V_{\dot{r} b^{*}}\end{array}$ & $\frac{4}{1}$ & $\frac{4}{1}$ & $\begin{array}{l}-4 \\
-1\end{array}$ & $\begin{array}{l}-4 \\
-1\end{array}$ & 1 \\
\hline$W_{r q}$ & $\frac{1}{2}$ & - & - & -1 & 1 \\
\hline$W_{r}$ & & - & - & & \\
\hline
\end{tabular}

Not averaged over reciprocal crosses:

$\begin{array}{llll}1 & - & - & - \\ \frac{1}{4} & \frac{1}{4} & - & -\frac{1}{4} \\ \frac{1}{4} & 1 & - & -1 \\ \frac{1}{4} & \frac{1}{4} & -\frac{1}{4} & -\frac{1}{4} \\ \frac{1}{4} & \frac{1}{4} & -\frac{1}{4} & -\frac{1}{4} \\ \frac{1}{2} & \frac{1}{4} & - & -\frac{1}{4} \\ \frac{2}{2} & - & - & -\frac{1}{4}\end{array}$

TABLE 6

Expectations of statistics from a diallel table

Method (b), sexes combined

Sex-linkage parameters

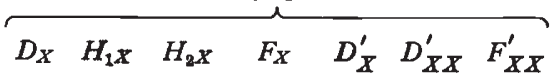

Autosomal parameters

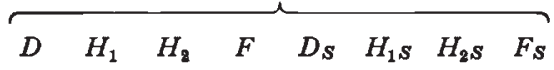

Averaged over reciprocals :

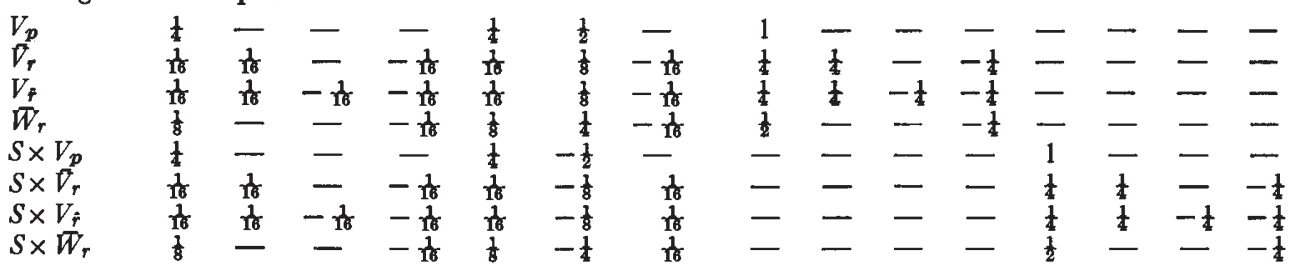

Not averaged over reciprocals:

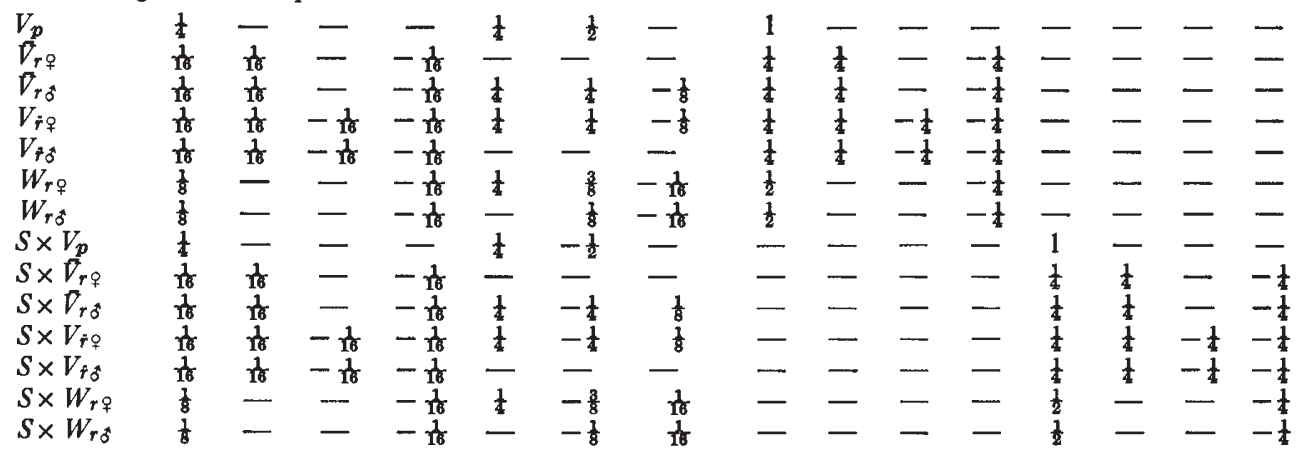


15 parameters are specified. Since the coefficient of $D_{X}$ is equal to that of $D_{X}^{\prime}$ in every statistic these two parameters cannot be separated, but even allowing for their combination separation of the remaining effects is impossible.

If the data are not averaged over reciprocal crosses, 14 statistics are available and there are no obvious correlations between parameters. Thus a solution is only possible if one parameter is omitted from the model. The particular parameter to be omitted will depend on the particular circumstances. Thus if gene frequencies are roughly equal or dominance is negligible the appropriate $F$ type parameters may be omitted or the $H_{1}$ and the $\mathrm{H}_{2}$ type parameters may be combined. If autosomal sex-limitation is absent, or complete, the autosomal parameters can be reduced. The decision as to which course of action is least damaging may be gauged by consideration of the analyses of variance described earlier. The assumptions made in estimating these parameters include absence of epistasis and correlated gene distributions. For autosomal genes it has been shown that failure of these assumptions could lead to distortion of the estimates obtained if either effect is very marked (Coughtrey and Mather, 1970).

Acknowledgments. - I am indebted to Dr M. J. Kearsey for his help and advice during the course of this work and for constructive criticism of the manuscript. The work was done whilst in receipt of a Science Research Council Studentship.

\section{REFERENCES}

ALlARD, R. W. 1956. The analysis of genetic environmental interactions by means of diallel crosses. Genetics, 41, 305-318.

COMSTOCK, R. E., AND ROBINSON, H. 1952. Estimation of average degree of dominance of genes. In Heterosis, pp. 494-516. Iowa State College Press.

COUGHTREY, A., AND MATHER, K. 1970. Interaction and gene association and dispersion in diallel crosses where gene frequencies are unequal. Heredity, 25, 79-88.

DICKINsON, A. G., AND JINKs, J. L. 1956. A generalised analysis of diallel crosses. Genetics, 41, 65-78.

DURRANT, A. 1965. Analysis of reciprocal differences in diallel crosses. Heredity, 20, 573-607.

HAYMAN, B. I. 1954. The analysis of variance of diallel tables. Biometrics, 10, 235-244.

JINKs, J. L. 1954. The analysis of continuous variation in a diallel cross of Nicotiana rustica. Genetics, 39, 767-788.

JINKS, J. L., AND BROADHURST, P. L. 1963. Diallel analysis of litter size and body weight in rats. Heredity, 18, 319-336.

KILLICK, R. J. 1971. Sex-linkage and sex-limitation in quantitative inheritance. I. Random mating populations. Heredity, 27, 175-188.

Mather, K., AND Jinks, J. L. 1971. Biometrical Genetics. Chapman and Hall, London.

TOPHAM, P. 1966. Diallel analysis involving maternal and maternal interaction effects. Heredity, 21, 665-674.

WEARDON, s. 1964. Alternative analyses of the diallel cross. Heredity, 19, 669-680. 